\title{
An Improved DV-HOP Localization Algorithm in Wireless Sensor Networks
}

\author{
Y.R. Wang, W. Quan, G.Q. Wang, Z.H. Qian \\ College of Communication Engineering \\ Jilin University \\ Changchun, Jilin, China
}

\begin{abstract}
According to the classical DV-HOP localization algorithm in wireless sensor networks with low precision, we put forward an improved algorithm,which adopted a new way to calculate the distance between unknown nodes and anchor nodes, then by using the weighted least squares method to calculate nodes coordinates. Matlab experimental simulation results show that under the same network environment, the algorithm can calculate the distance to reduce localization errors effectively, and improve the localization accuracy.
\end{abstract}

Keywords-wireless sensor networks; node localization; DV-HOP algorithm

\section{INTRODUCTION}

In wireless sensor networks (WSN), monitoring information without location information are meaningless, so determine the position of the nodes are the basic function of WSN. But the sensor nodes are limited by the cost of energy and volume, we can't equip each node with GPS moudle. So the research is how to use a small amount of anchor nodes to work out the precise position[1-2]. In recent years, research results show that the localization algorithm can be divided into two categories:Base on the distance algorithm and range free localization algorithm, the latter with low hardware requirements and low energy cost and get more attention. Especially, DV-HOP algorithm is the most representative algorithm. But DV-HOP algorithm has defects of low localization accuracy, therefore,we proposed an improved algorithm, import the new concept of distance. At last, through the simulation experiments are performed to verify the localization precision and stability of improved algorithm. The basic idea of DV-HOP algorithm is represent the distance between unknown nodes and anchor nodes as the product of hops and the average jumped distance. Specific process is as follows: At first, Network nodes broadcast the anchor nodes' location information, so all nodes obtain the smallest hops to anchor nodes. Then calculate the average jumped distance and broadcast to the whole net.

$$
\text { HopSize }_{i}=\frac{\sum_{i \neq j} \sqrt{\left(x_{i}-x_{j}\right)^{2}+\left(y_{i}-y_{j}\right)^{2}}}{\sum_{j \neq i} h_{j}}
$$

Among them, $\left(\mathrm{X}^{\mathrm{i}}, \mathrm{Y}^{\mathrm{i}}\right),\left(\mathrm{X}_{\mathrm{j}}, \mathrm{Y}^{\mathrm{j}}\right)$ is the coordinates of anchor nodes, $i, j$ and $\mathrm{H}^{j}$ is the hop number between $i$ and $j(i \neq j)$. The unknown nodes according to the hop number and the jumped distance received from the nearest anchor node to calculate the
Euclidean distance to each anchor node. At last, if the unknown nodes obtain 3 or more than 3 anchor nodes hop distance, using three edge measurement formula (2) to calculate their coordinates :

$$
\left\{\begin{array}{c}
\left(x_{1}-x\right)^{2}+\left(y_{1}-y\right)^{2}=d_{1}^{2} \\
\vdots \\
\left(x_{n}-x\right)^{2}+\left(y_{n}-y\right)^{2}=d_{n}^{2}
\end{array}\right.
$$

\section{THE IMPROVED DV-HOP ALGORITHM}

The errors about original algorithm mainly from the average hop distance. Literature[3] use the minimum mean square error criterion to calculate the average hop distance. Literature[4] put forward a kind of method based on weighted processing to calculate the average hop distance. The methods of the above literatures although can improve the accuracy to a certain extent, but there are still some problems. According to the calculate process of the DV-HOP localization algorithm, we improved the estimation from the distance between unknown nodes to anchor nodes. As shown in Figure 1, assuming that the nearest anchor node to the unknown node $\mathrm{C}$ is $\mathrm{A}$, what we need to do is to estimate the distance from unknown node $\mathrm{C}$ to the anchor node $\mathrm{B}$, which is named $\mathrm{d}$ ${ }_{B C}$.Under the case of non-collinear in $A B C, d^{A C} d^{A B} d^{B C}$ will constitute a triangle. Because the hop number between nodes constraint of communication radius, it will have a strong fault tolerance under normal circumstances. If the node path does not appear twists and turns, we can use the length of the jump measure the distance approximately measure. So under the case of we know the hop number between $\mathrm{AB}, \mathrm{AC}, \mathrm{BC}$, we can use the hop number of the trilateral to represent the distance of the trilateral

$$
\cos \alpha \approx \frac{H_{1}^{2}+H_{2}^{2}-H_{3}^{2}}{2 H_{1} H_{2}}
$$

Among them, $\mathrm{H}^{1}$ is the hop number between anchor node $\mathrm{A}$ and unknown nodes, $\mathrm{H}^{2}$ is the hop number between anchor node $\mathrm{A}$ and anchor node $\mathrm{B}, \mathrm{H}^{3}$ is the hop number between anchor node B and unknown nodes, all of them are known as value. 


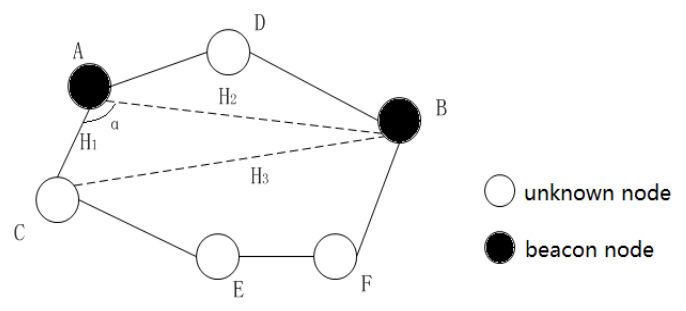

FIGURE I. AN EXAMPLE OF DISTANCE ESTIMATION

The distance between the anchor node $A B$ is $d^{A B}$ which is known as the exact value. The hop number between node $\mathrm{A}$ and $\mathrm{C}$ is small, the distance between $\mathrm{A}$ and $\mathrm{C}$ can be work out accurately through the product of the average hop distance and hop number of anchor node A which named $\mathrm{d}^{\mathrm{AC}}$, then we can get the estimation of $\mathrm{dBC}$ through the next formulas :

$$
\begin{gathered}
d_{A C} \approx \text { HopSize }_{A} \times H_{1} \\
d_{B C}=\sqrt{d_{A C}^{2}+d_{A B}^{2}-\frac{H_{1}^{2}+H_{2}^{2}-H_{3}^{2}}{H_{1} H_{2}} d_{A C} d_{A B}}
\end{gathered}
$$

Among them, HopSize ${ }^{\text {e }}$ is the average hop distance of anchor node A through formula (1). In fact, the trilateral calculation method in this paper use the accurate value $\mathrm{d}^{\mathrm{AB}}$ to correct the final result and make it conform to the real situation. When the hop distance of the trilateral can't compose the triangle, we adopt formula (6) to calculate the distance :

$$
d_{B C} \approx \text { HopSize }_{A} \times H_{3}
$$

Then we use the maximum likelihood estimation to calculate the coordinates, get the linear equation such as formula (7) :

$$
\begin{aligned}
& A X=B \\
& A=2\left[\begin{array}{cc}
x_{1}-x_{n} & y_{1}-y_{n} \\
\vdots & \vdots \\
x_{n-1}-x_{n} & y_{n-1}-y_{n}
\end{array}\right] \\
& B=\left[\begin{array}{c}
x_{1}^{2}-x_{n}^{2}+y_{1}^{2}-y_{n}^{2}+d_{n}^{2}-d_{1}^{2} \\
\vdots \\
x_{n-1}^{2}-x_{n}^{2}+y_{n-1}^{2}-y_{n}^{2}+d_{n}^{2}-d_{n-1}^{2}
\end{array}\right] X=\left[\begin{array}{l}
x \\
y
\end{array}\right]
\end{aligned}
$$

Use minimum variance estimation standard methods can get the coordinates of unknown nodes as formula (8) :

$$
\hat{X}=\left(A^{T} A\right)^{-1} A^{T} B
$$

\section{THE SIMULATION EXPERIMENT}

In order to verify the validity and feasibility of this algorithm,we simulate the experiment of the improved DV-HOP algorithm and the algorithm in literature [5], then compare and analyze the results between them. The simulation worked in Matlab2012, distributed 300 nodes randomly in 150 $\mathrm{m} * 150 \mathrm{~m}$ region. Part of them are anchor nodes, carrying the GPS equipment which can get their own coordinates, and the others are unknown nodes which need to calculate the coordinates. All nodes have the whole network of unique ID number and relatively static. The radius of node communication refer experience value.Therefore, set for the node communication radius as $15 \mathrm{~m}$ and $30 \mathrm{~m}$, observe the 3 algorithms in the case of low and high node connectivity degree. Figure 2 and figure 3 set the node communication radius as $15 \mathrm{~m}$ and $30 \mathrm{~m}$, each kind of simulation algorithm for random operation 100 times, finally take the average. The calculation method of node relative positioning error is as follows :

$$
\text { error }_{i}=\frac{\sqrt{\left(x_{i}-x_{i}^{\prime}\right)^{2}+\left(y_{i}-y_{i}^{\prime}\right)^{2}}}{R} \times 100 \%
$$

Among them, $\left(\mathrm{x}^{\mathrm{i}}, \mathrm{y}^{\mathrm{i}}\right)$ is true coordinates of unknown node $\mathrm{i}$, $\left(\mathrm{x}_{\mathrm{i}}^{\prime}, \mathrm{y}_{\mathrm{i}}^{\prime}\right)$ is the estimate coordinates of unknown node $\mathrm{i}, \mathrm{R}$ is communication radius.

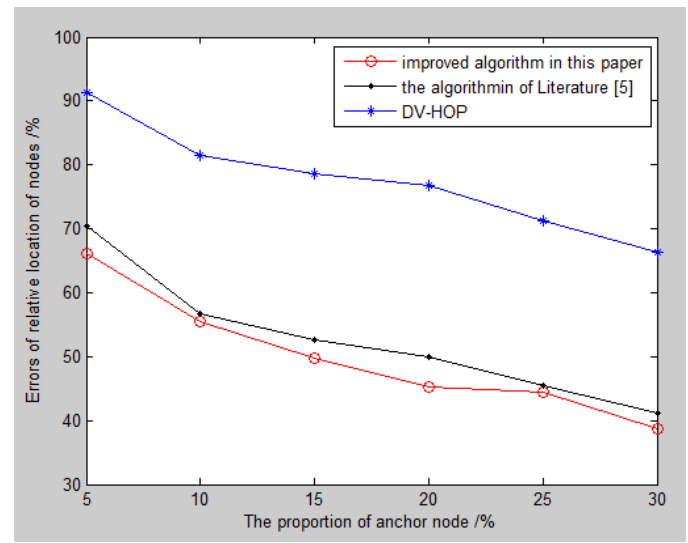

FIGURE II. LOCALIZATION ERROR VARYING RADIO OF ANCHORS WHEN $=15 \mathrm{M}$

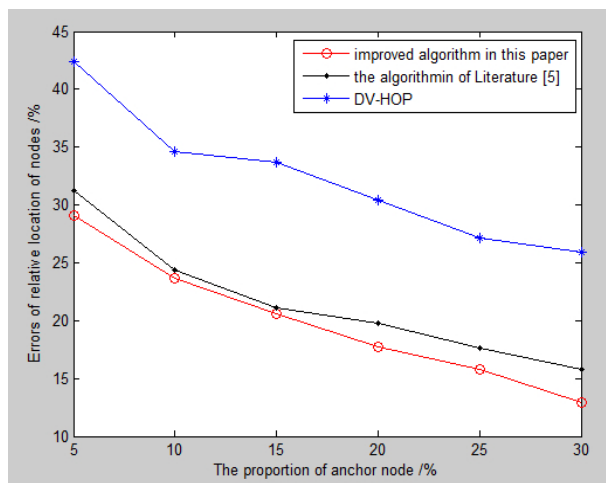

FIGURE III. LOCALIZATION ERROR VARYING RADIO OF ANCHORS WHEN $=30 \mathrm{M}$ 


\section{CONCLUSION}

Because of the accuracy is not high in DV-HOP algorithm, we put forward a new improved algorithm to calculate the coordinates. According to the triangle formed from the unknown node and two anchor nodes, use the hop number and the distance between anchor nodes to estimate the distance from unknown node to anchor nodes. Then use the least square method to estimates the unknown nodes' coordinates. From the results of the simulation,the improved algorithm in this paper have obvious advantage about accuracy and the stability of nodes. In the further, the work will study how to make the improved algorithm to improve the positioning accuracy and reduce the communication and computation,so as to minimize the energy consumption of nodes and improve the algorithm's practical value, it will be a very meaningful and worthwhile research topic.

\section{REFERENCES}

[1] Akyildiz I, Su W, Sankarasubramaniam Y,et al. A survey on sensor network [J]. IEEECommunications Magazine, 40 (8) : 102 - 114, 2002.

[2] Niclescu D, Americ N L. Communicartion paradigms for sensor networks [J]. IEEE Communications Magazine, 43 (3) : 116 - 122 , 2005.

[3] W.W.Ji, Z.Liu. Study on application of DV- HOP localization algorithms to random sensor networks [J]. Journal of Electronics \& Information Technology, 30 (4) : 970 - 974, 2005.

[4] X.S.Wang, Y.J.Zhao, H.T.Li.Improved study based on DV-HOP localization algorithm [J]. Computer Science, 38 (2) : 76 - 78 , 2011.

[5] M.Y.Shen, Y.Zhang. DV- HOP localization algorithm based on improved average hop distance and estimate of distance [J]. Application Research of Computers, 28 (2) : 648 - $650,2011$. 